\title{
Consistent Total Traction Torque-Oriented Coordinated Control of Multimotors with Input Saturation for Heavy-Haul Locomotives
}

\author{
Changfan Zhang, ${ }^{1}$ Qian Zhang $\mathbb{C}^{1},{ }^{1}$ Jing He, ${ }^{1}$ Jianhua Liu ${ }^{1},{ }^{1}$ Xingxing Yang, \\ and Songan $\mathrm{Mao}^{2}$ \\ ${ }^{1}$ College of Electrical and Information Engineering, Hunan University of Technology, Zhuzhou 412007, China \\ ${ }^{2}$ School of Electrical and Computer Engineering, Purdue University, West Lafayette, IN 47907, USA \\ Correspondence should be addressed to Jianhua Liu; jhliu@hut.edu.cn
}

Received 25 October 2019; Revised 29 September 2020; Accepted 10 October 2020; Published 30 October 2020

Academic Editor: Maria C. Falvo

Copyright (c) 2020 Changfan Zhang et al. This is an open access article distributed under the Creative Commons Attribution License, which permits unrestricted use, distribution, and reproduction in any medium, provided the original work is properly cited.

\begin{abstract}
In the coordinated control of multiple motors for heavy-haul locomotives, the input value for a motor often exceeds its maximum allowable input value, resulting in the saturation problem. A traction total-amount coordinated tracking control (TACTC) strategy is proposed to address the input saturation of heavy-haul locomotives driven by multiple motors. This strategy reduces control input and suppresses input saturation. First, a multimotor traction model with uncertain parameter perturbations and external disturbances was established. Next, a sliding-mode disturbance observer (SMDO) was designed to reduce the slidingmode switching gain, thereby decreasing the control input. An auxiliary anti-windup (AW) system was used to weaken the effect of input saturation on tracking performance. Then, the observed value and auxiliary state were fed back to the sliding-mode controller to design a TACTC protocol and ensure that the total amount of traction torque follows the desired traction characteristic curve. Finally, the Matlab/Simulink simulation and RT-Lab semiphysical experiment results show that the proposed strategy can effectively suppress the input saturation problem of multimotor coordinated control.
\end{abstract}

\section{Introduction}

Multiple motors together providing traction torque is a common traction-drive method used in heavy-haul locomotives. The complex and changeable running environment of locomotives often causes the loss of traction performance of the motors. The traction system can be safely and smoothly operated only when the total amount of traction torque provided by the multiple motors is consistent with the desired total amount $[1,2]$. This condition implies the maximum utilization of the efficiency for multiple motors [3]. However, the saturation problem that the input value required for a motor exceeds its maximum allowable value often occurs in practical engineering. Input saturation is a nonlinear problem that affects the dynamic performance of the system, makes the system unstable, and even damages the device $[4,5]$.
Input saturation problems are common in the coordinated control of multiple motors based on the theory of total traction torque consistency. These problems seriously affect the overall traction performance of the locomotive and cause motor failure in severe cases. The word "saturation" originates from the integrator in proportional-integral control [6]. Kothare et al. [7] proposed a unified framework for traditional anti-windup. Subsequently, Tarbouriech et al. $[8,9]$ further developed modern anti-windup (AW) technology. In recent years, the modern AW compensator has been used as an effective measure to suppress input saturation [10-15]. A combination of a Nussbaum function, smooth function, or mean value theorem is a common method to address input saturation [16-18], and by using the linear matrix inequality (LMI) to solve the parameters of the AW compensator, the estimation of the domain of attraction 
can be improved $[19,20]$. In [21], the AW compensator synthesis scheme was derived by employing the quadratic Lyapunov function, notion of quadratic inner-boundedness, one-sided Lipschitz condition, sector condition, and $L_{2}$ gain minimization. In [22], an adaptive neural network controller with an integrated auxiliary system is designed to guarantee the tracking performance and tackle the effect of actuator saturation. Furthermore, in [23], the disturbance was cancelled by increasing the sliding-mode switching gain; although this process reduces the complexity of the system, it increases the control input and may cause input saturation. Thus, it appears that the literature contains no reports on the input saturation of consistent total traction torque-oriented multimotor coordination. Therefore, based on an actual system of heavy-haul locomotives, a total-amount coordinated tracking control framework was constructed in this study. This framework addresses the input saturation, considering the SMDO and auxiliary AW system.

The basic idea of this framework was presented in $[1,2]$. This work presents great improvement over the previous works: the problem of input saturation in coordinated control of multiple motors is solved, and the theory of totalamount consistency is improved. The specific innovations are as follows: (1) The SMDO was used to reconstruct the parameter perturbation and load torque disturbance to avoid input saturation, which is caused by an increase in the sliding-mode switching gain. (2) To directly compensate for the control input, an auxiliary AW system was constructed. The state of the auxiliary system was introduced into the design of the controller to effectively suppress the influence of input saturation on the overall traction performance of multiple motors.

The rest of this paper is organized as follows: The next section describes the construction of a TACTC framework. The third section presents the design of a multimotor traction model, and the SMDO as well as a stability analysis. Subsequently, the use of the auxiliary AW system to design the controller with input saturation is described along with its convergence analysis in Section 4 . The simulation and experimental results are then provided to validate the effectiveness of the observer and controller in Section 5. Finally, the conclusions of the work are presented in Section 6.

\section{System Framework}

In the multiaxis coordinated control of multiple motors for heavy-haul locomotives, the expectation is not to make the individual states consistent (in terms of parameters such as the speed, position, and torque) but to ensure that the total amount of traction torque provided by the multiple motors is consistent with the desired traction characteristic curve during the dynamic adjustment of the rail surface characteristics. Thus, the total traction torque amount of the locomotive can be maintained. In this special context, the saturation problem of multiple motors has become increasingly serious and complicated. Therefore, a TACTC framework of heavy-haul locomotives with input saturation, as shown in Figure 1, was constructed in this study. The system framework comprises the control module, the drive module, and the motor module. The main aim of this study was to design a TACTC strategy that combines the SMDO and auxiliary AW technology, as shown in the control module. When multiple motors coordinate the traction torque output redundancy of each individual motor, the input value of a motor often exceeds its maximum allowable input value. To weaken the effect of input saturation on tracking performance, a TACTC strategy was designed. In the drive module, the control signals, obtained using the proposed TACTC strategy, generate PWM signals with corresponding properties via the pulse width modulation (PWM) technology to control the ON/OFF state of the transistor in the drive circuit to drive the motor [24-26].

The multimotor system coordinates the torque output of each motor through the TACTC such that the total amount of traction torque tends to be consistent with the desired traction characteristic curve within a finite time $t_{s}$ :

$$
\lim _{t \longrightarrow t_{s}}\left(\sum_{j=1}^{m} T_{e j}-T^{*}\right)=0,
$$

where $T^{*}$ is the desired traction characteristic curve, and $T_{e j}$ denotes the $j^{\text {th }}$ motor output torque.

\section{Mathematical Model and Observer Design}

3.1. Mathematical Model of Multimotors with Disturbance. In this work, the multimotor traction system is based on the DC permanent magnet motor mathematical model (2), which is obtained by applying Kirchoff's voltage law to an armature electric circuit and Newton' laws to the mechanical subsystem [27-29]. The schematic diagram is shown in Figure 2. The $j^{\text {th }}$ motor is taken as an example. $R_{j}$ and $L_{j}$ represent the resistance and inductance of the armature circuit, respectively. Further, $i_{j}$ is the armature current; $\omega_{j}$, the output angular velocity of the motor gearbox; $u_{j}$, the voltage of the input terminals of the armature circuit; $k_{e j}$, the back electromotive force constant; $k_{t j}$, the speed reduction ratio of the gearhead; $J$, the inertia moments $\left(J_{0 j}\right.$ and $J_{1 j}$ represent the inertia moments of the motor and gearhead, respectively); $b$, the viscous damping coefficient $\left(b_{0 j}\right.$ and $b_{1 j}$ indicate the viscous damping coefficients of the motor and the gearhead, respectively); $k_{m j}$, the motor torque constant; and $T_{L j}$, the load torque.

Given a multimotor traction system consisting of $m$ motors, the voltage and torque balance equations [30] of the $j^{\text {th }}$ motor are as follows: 


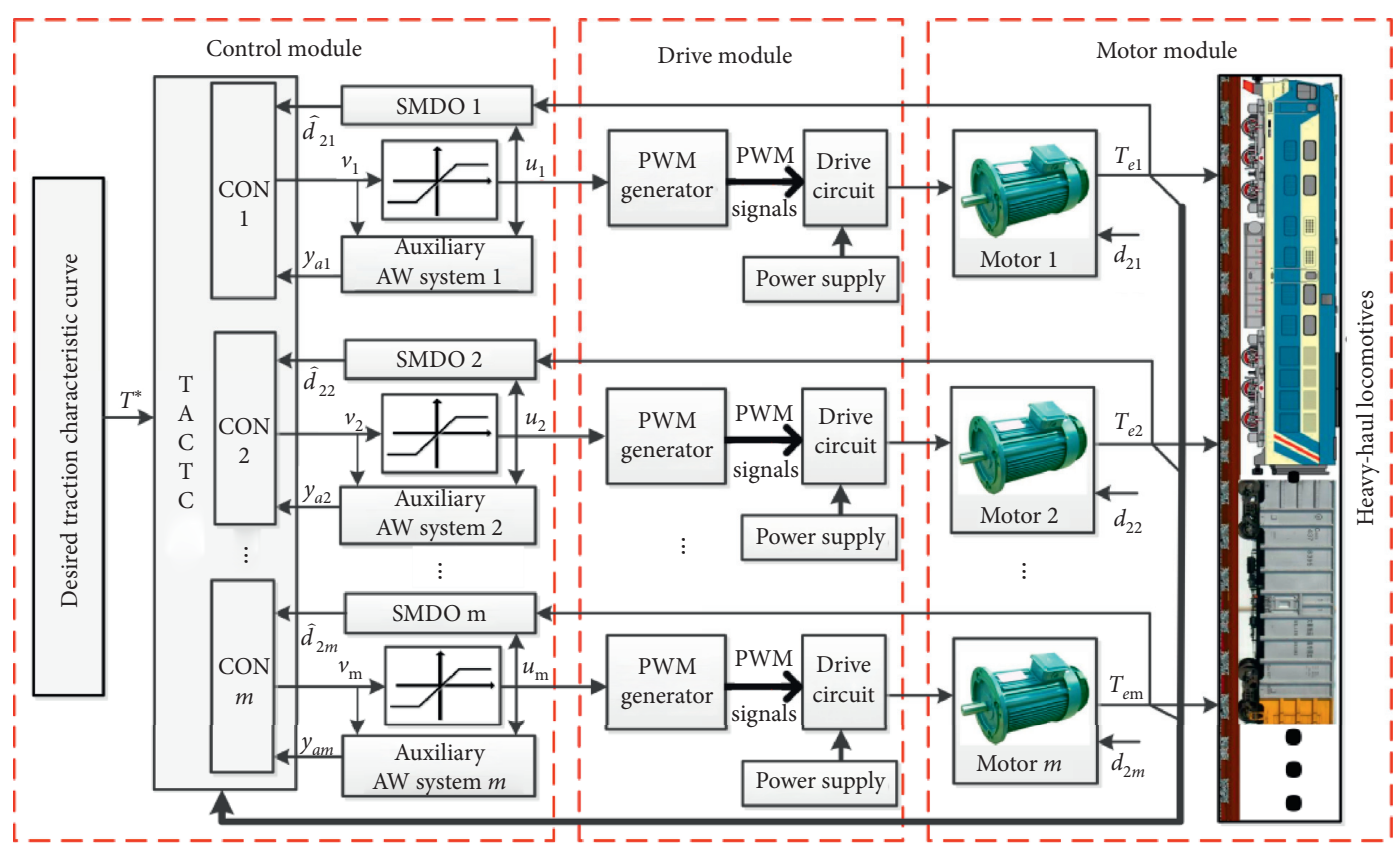

FIgURE 1: The system framework.

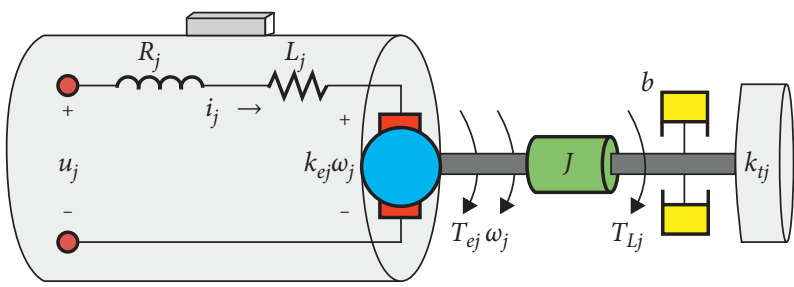

Figure 2: Schematic diagram of the DC permanent magnet motor with gearhead (reproduced from F. Beltran-Carbajal et al.'s "An asymptotic differentiation approach of signals in velocity tracking control of DC motors", Electric Power Systems Research, 2015 (122) 218-223, Under the Creative Commons Attribution License/public domain).

$$
\left\{\begin{array}{l}
L_{j} \frac{\mathrm{d} i_{j}}{\mathrm{~d} t}=-R_{j} i_{j}-k_{e j} k_{t j} \omega_{j}+u_{j}, \\
\left(J_{1 j}+k_{t j}^{2} J_{0 j}\right) \frac{\mathrm{d} \omega_{j}}{\mathrm{~d} t}=-\left(b_{1 j}+k_{t j}^{2} b_{0 j}\right) \omega_{j}+T_{e j}-T_{L j}, \quad(j=1,2, \ldots, m), \\
T_{e j}=k_{t j} k_{m j} i_{j j} .
\end{array}\right.
$$

Given that the output angular velocity is differentially flat, all variables and control inputs, including the electric current, can be represented by the output angular velocity and its derivative [31]. Thus, the output angular velocity is the state variable, and the following equation is obtained by eliminating term $i_{j}$ from equation (2):

$$
\ddot{x}_{1 j}+a_{1 j} \dot{x}_{1 j}+a_{0 j} x_{1 j}=b_{j} u_{j}+f_{j},
$$

where $\quad\left(a_{1 j}=\left(b_{\text {eq } j} / J_{\text {eqj }}\right)+\left(R_{j} / L_{j}\right)\right), \quad\left(a_{0 j}=\left(k_{t j}^{2} k_{m j} k_{e j}+\right.\right.$ $\left.\left.R_{j} b_{\text {eq } j} / J_{\text {eq } j} L_{j}\right)\right), \quad\left(b_{j}=\left(k_{t j} k_{m j} / J_{\text {eq } j} L_{j}\right)\right), \quad$ and
$\left(f_{j}=-\left(R_{j} / J_{\text {eq } j} L_{j}\right) T_{L_{j}}-\left(1 / J_{\text {eq } j}\right) \dot{T}_{L_{j}}\right)$. Here, $f_{j}$ is the unknown time-varying disturbance; $\left(J_{\text {eq } j}=J_{1 j}+k_{t j}^{2} J_{0 j}\right)$, the equivalent moment of inertia; and $\left(b_{\mathrm{eq} j}=b_{1 j}+k_{t j}^{2} b_{0 j}\right)$, the equivalent viscous damping constant.

Let $x_{1 j}=\omega_{j}, x_{2 j}=\dot{x}_{1 j}$ and $x_{3 j}=T_{e j}$. Given that the parameters of the motor vary with time during operation (For example, the resistance, inductance, and other parameters change with temperature.), the parameter perturbation and load torque disturbance are uniformly attributed to unknown compound disturbances. Then, the state equation can be expressed as follows: 


$$
\dot{x}_{j}=\bar{A}_{j} x_{j}+\bar{B}_{j} u_{j}+d_{j},
$$

where $\quad x_{j}=\left(\begin{array}{c}x_{1 j} \\ x_{2 j} \\ x_{3 j}\end{array}\right), \quad \bar{A}_{j}=\left(\begin{array}{ccc}0 & 1 & 0 \\ -\bar{a}_{0 j} & -\bar{a}_{1 j} & 0 \\ -\bar{J}_{\mathrm{eq} j} \bar{a}_{0 j} & \bar{b}_{\mathrm{eq} j}-\bar{J}_{\mathrm{eq} j} \bar{a}_{1 j} & 0\end{array}\right)$, $\bar{B}_{j}=\left(\begin{array}{c}0 \\ \bar{b}_{j} \\ \bar{J}_{\mathrm{eq} j} \bar{b}_{j}\end{array}\right), u_{j}=\left(\begin{array}{c}0 \\ u_{j} \\ u_{j}\end{array}\right)$, and $d_{j}=\left(\begin{array}{c}0 \\ d_{1 j} \\ d_{2 j}\end{array}\right)$. Here, $d_{1 j}=\Delta a_{0 j} x_{1 j}+\Delta a_{1 j} x_{2 j}+\Delta b_{j} u_{j}+f_{j}(t), \quad d_{2 j}=\Delta J_{\mathrm{eqj}} a_{0 j} x_{1 j}$ $+\Delta b_{\text {eq } j} x_{2 j}-\Delta J_{\text {eq } j} a_{1 j} x_{2 j}+\Delta J_{\text {eq } j} b_{j} u_{j}+J_{\text {eq } j} f_{j}(t)+\dot{T}_{L_{j}}$.

3.2. Observer Design Based on Sliding-Mode. In this study, the parameter perturbation and load torque disturbance are uniformly attributed to an unknown compound disturbance $d_{2 j}$ that is estimated by using the measurable state variable $x_{1 j}$ and output $x_{3 j}$. The sliding-mode observer can be constructed from equation (4) as follows:

$$
\dot{\hat{x}}_{j}=\bar{A}_{j} \widehat{x}_{j}+\bar{B}_{j} u_{j}+w_{j},
$$

where $\widehat{x}_{j}=\left[\begin{array}{lll}\hat{x}_{1 j} & \hat{x}_{2 j} & \hat{x}_{3 j}\end{array}\right]^{\mathrm{T}}$ is an estimated value of the state; $w_{j}=\left[\begin{array}{lll}k_{1} \operatorname{sgn}\left(e_{1}\right) & 0 & k_{3} \operatorname{sgn}\left(e_{3}\right)\end{array}\right]^{\mathrm{T}} ; \operatorname{sgn}()$ represents the sign function; and $k_{1}$ and $k_{3}$ are the positive constants to be designed. The estimated compound disturbance is defined as $\widehat{d}_{2 j}$. The estimated error of the state is $e_{i}=x_{i j}-\widehat{x}_{i j}$, $i=1,2,3$, with the maximum error $\left|e_{i}\right|_{\max }$ and error matrix $E_{j}=\left[\begin{array}{ll}e_{1} & e_{3}\end{array}\right]^{\mathrm{T}}$.

Assumption 1. The unknown compound disturbance $d_{2 j}$ is bounded; that is, there is a positive constant $\left|d_{2 j}\right|_{\max }$ such that $d_{2 j} \leq\left|d_{2 j}\right|_{\max }$.

Theorem 1. For the multimotor system (4), the SMDO is designed as shown in equation (5). When the parameter satisfies $k_{1}>\left|e_{2}\right|_{\max }+\eta_{1}$ and $k_{3}>\left|d_{2 j}\right|_{\max }+\eta_{3}$, where $\eta_{1}$ and $\eta_{3}$ are any positive constants, the observer error converges to the sliding-mode surface within a finite time $T_{1}$, and the estimated value of the disturbance is $\lim _{t \rightarrow T_{1}} \widehat{d}_{2 j}=d_{2 j}=k_{3}$ $\operatorname{sgn}\left(x_{3 j}-\hat{x}_{3 j}\right)-\left(\bar{b}_{e q j}-\bar{J}_{e q j} \bar{a}_{1 j}\right) k_{1} \operatorname{sgn}\left(x_{1 j}-\hat{x}_{1 j}\right)$.

Proof. Select the sliding-mode surface as follows:

$$
s_{1}=E_{j} .
$$

By deriving and subtracting (5) from (4), the following is obtained:

$$
\begin{aligned}
\dot{s}_{1} & =\dot{E}_{j}=\left[\begin{array}{l}
\dot{e}_{1} \\
\dot{e}_{3}
\end{array}\right] \\
& =\left[\begin{array}{c}
e_{2}-k_{1} \operatorname{sgn}\left(e_{1}\right) \\
-\bar{J}_{\mathrm{eq} j} \bar{a}_{0 j} e_{1}+\left(\bar{b}_{\mathrm{eq} j}-\bar{J}_{\mathrm{eqj}} \bar{a}_{1 j}\right) e_{2}+d_{2 j}-k_{3} \operatorname{sgn}\left(e_{3}\right)
\end{array}\right] .
\end{aligned}
$$

Here, note that equation (7) is a differential equation containing the sign function of discontinuous terms on the right-hand side of the equation. The differential equation can be solved by using the Filippov theorem [32], which can ensure that the state estimated error $e_{i}(i=1,2,3)$ is bounded.

A positive definite Lyapunov function is selected as follows:

$$
V_{1}=\frac{1}{2} E_{j}^{T} E_{j}
$$

From $V_{1}$, the following is obtained:

$$
\begin{aligned}
\dot{V}_{1}= & E_{j}^{T} \dot{E}_{j}=\left[\begin{array}{ll}
e_{1} & e_{3}
\end{array}\right]\left[\begin{array}{ll}
\dot{e}_{1} & \dot{e}_{3}
\end{array}\right]^{\mathrm{T}} \\
\leq & \left|e_{1}\right|\left|e_{2}\right|-k_{1}\left|e_{1}\right|-\bar{J}_{\mathrm{eq} j} \bar{a}_{0 j}\left|e_{3}\right|\left|e_{1}\right| \\
& +\left(\bar{b}_{\mathrm{eq} j}-\bar{J}_{\mathrm{eq} j} \bar{a}_{1 j}\right)\left|e_{3}\right|\left|e_{2}\right|+\left|e_{3}\right|\left|d_{2 j}\right|-k_{3}\left|e_{3}\right| \\
\leq & \left|e_{1}\right|\left[\left|e_{2}\right|-k_{1}\right]+\left|e_{3}\right|\left[\left|d_{2 j}\right|-k_{3}\right] \\
\leq & -\min \left\{k_{1}-\left|e_{2}\right|, k_{3}-\left|d_{2 j}\right|\right\}\left\|E_{j} \mid\right\| .
\end{aligned}
$$

If the design parameters satisfy $k_{1}>\left|e_{2}\right|_{\max }+\eta_{1}$ and $k_{3}>\left|d_{2 j}\right|_{\max }+\eta_{3}$, where $\eta_{1}>0$ and $\eta_{3}>0$, then

$$
\dot{V}_{1} \leq-\min \left\{\eta_{1}, \eta_{3}\right\}\left\|E_{j}\right\|=-\eta\left\|E_{j}\right\|,
$$

where $\eta=\min \left\{\eta_{1}, \eta_{3}\right\}$. Therefore, $\dot{V}_{1} \leq 0$ can be obtained if and only if $e_{1} \longrightarrow 0$ and $e_{3} \longrightarrow 0$, which causes $V_{1} \longrightarrow 0$. Thus, SMDO equation (5) is globally asymptotically stable. Based on the assumption that $T_{1}$ is the time needed to reach the sliding-mode surface, $T_{1}$ is a finite value. When the sliding-mode surface is reached $\left(t>T_{1}\right)$, the principle of sliding-mode equivalence [33] can be applied to equation (7). Then, the following holds:

$$
\begin{gathered}
\left\{\begin{array} { l } 
{ s _ { 1 } = 0 } \\
{ \dot { s } _ { 1 } = 0 }
\end{array} \Rightarrow \begin{array} { l } 
{ e _ { 1 } = 0 } \\
{ \dot { e } _ { 1 } = 0 }
\end{array} \Rightarrow \left\{\begin{array}{l}
x_{1 j}=\hat{x}_{1 j}, \\
e_{2}=k_{1} \operatorname{sgn}\left(e_{1}\right)=k_{1} \operatorname{sgn}\left(x_{1 j}-\widehat{x}_{1 j}\right),
\end{array}\right.\right. \\
\left\{\begin{array} { l } 
{ e _ { 3 } = 0 } \\
{ \dot { e } _ { 3 } = 0 }
\end{array} \Rightarrow \left\{\begin{array}{l}
x_{3 j}=\widehat{x}_{3 j}, \\
d_{2 j}=k_{3} \operatorname{sgn}\left(e_{3}\right)-\left(\bar{b}_{\mathrm{eq} j}-\bar{J}_{\mathrm{eq} j} \bar{a}_{1 j}\right) e_{2} .
\end{array}\right.\right.
\end{gathered}
$$

Therefore, the unknown compound disturbance can be reconstructed as follows:

$$
\begin{aligned}
\lim _{t \longrightarrow T_{1}} \hat{d}_{2 j}= & d_{2 j}=k_{3} \operatorname{sgn}\left(x_{3 j}-\widehat{x}_{3 j}\right) \\
& -\left(\bar{b}_{\text {eqj }}-\bar{J}_{\text {eqj }} \bar{a}_{1 j}\right) k_{1} \operatorname{sgn}\left(x_{1 j}-\widehat{x}_{1 j}\right) .
\end{aligned}
$$

That is, $\forall \zeta_{j}>0$ and $\exists t_{1}<T_{1}$. If $t>t_{1}$, then

$$
\left|d_{2 j}-\widehat{d}_{2 j}\right|<\zeta_{j}
$$

In general, the discontinuity of the sign function can lead to the chattering phenomenon in the system. To suppress chattering, a continuous function $(F=(s /(|s|+$ $\sigma))$ ) is used instead of the sign function, where $\sigma$ is a small positive constant, and $s$ is the sliding-mode surface. 


\section{Controller Design with Input Saturation}

An auxiliary AW system [34] is used for the $j^{\text {th }}$ motor to reduce the influence of input saturation on the tracking performance of the multiple motors.

$$
\begin{cases}\dot{x}_{a j}= \begin{cases}-A_{a j} x_{a j}-\frac{\left|s_{2} B_{a j} \Delta u\right|+0.5 \Delta u^{2}}{\left|x_{a j}\right|}+\Delta u, & \left|x_{a j}\right| \geq \tau, \\ 0, & \left|x_{a j}\right|<\tau,\end{cases} \\ y_{a j}=x_{a j},\end{cases}
$$

where $x_{a j}$ is the auxiliary state; $y_{a j}$, the system output; $A_{a j}$, a positive coefficient to be designed; $\tau$, a small positive constant. Further, $\Delta u=u_{j}-v_{j}$, and $s_{2}$ is the sliding-mode surface, and $B_{a j}>\bar{J}_{\mathrm{eq} j} \bar{b}_{j}>0$ is a constant.

The tracking error between the total-amount of traction torque and the desired traction characteristic curve is

$$
e=\sum_{j=1}^{m}\left(x_{3 j}\right)-T^{*} \text {. }
$$

From equation (15), we get the following:

$$
\begin{aligned}
\dot{e}= & \sum_{j=1}^{m}\left(\dot{x}_{3 j}\right)-\dot{T}^{*}=\sum_{j=1}^{m}\left[-\bar{J}_{\mathrm{eq} j} \bar{a}_{0 j} x_{1 j}\right. \\
& \left.+\left(\bar{b}_{\mathrm{eq} j}-\bar{J}_{\mathrm{eq} j} \bar{a}_{1 j}\right) x_{2 j}+\bar{J}_{\mathrm{eq} j} \bar{b}_{j} u_{j}+d_{2 j}\right]-\dot{T}^{*} .
\end{aligned}
$$

The sliding-mode surface is selected as follows:

$$
s_{2}=e
$$

Then, substituting it into equation (16) yields the following:

$$
\begin{aligned}
\dot{s}_{2}= & \dot{e}=\sum_{j=1}^{m}\left[-\bar{J}_{\mathrm{eq} j} \bar{a}_{0 j} x_{1 j}+\left(\bar{b}_{\mathrm{eq} j}-\bar{J}_{\mathrm{eq} j} \bar{a}_{1 j}\right) x_{2 j}\right. \\
& \left.+\bar{J}_{\mathrm{eq} j} \bar{b}_{j} u_{j}+d_{2 j}\right]-\dot{T}^{*} .
\end{aligned}
$$

Thus, the estimated value of the compound disturbance and the auxiliary state are introduced, and the TACTC based on the sliding mode is designed as follows:

$$
\begin{aligned}
v_{j}= & \frac{1}{\bar{b}_{j}}\left[\bar{a}_{0 j} x_{1 j}-\left(\frac{\bar{b}_{\mathrm{eq} j}}{\bar{J}_{\mathrm{eq} j}}-\bar{a}_{1 j}\right) x_{2 j}-\frac{\widehat{d}_{2 j}}{\bar{J}_{\mathrm{eq} j}}-\frac{c_{2 j}}{\bar{J}_{\mathrm{eq} j}}\left(s_{2}-x_{a j}\right)\right. \\
& \left.-\frac{\varepsilon_{j}}{\bar{J}_{\mathrm{eq} j}} \operatorname{sgn}\left(s_{2}\right)\right]+\frac{1}{m \bar{J}_{\mathrm{eq} j} \bar{b}_{j}} \dot{T}^{*} .
\end{aligned}
$$

where $c_{2 j}$ and $\varepsilon_{j}$ are the positive constants to be designed, $j=1,2, \ldots, m$.
Theorem 2. For the multimotor traction system in equation (4), under the influence of the SMDO in equation (5) and the auxiliary $A W$ system in equation (14), the TACTC based on the sliding mode is given as shown in equation (19). When the parameters satisfy $0<c_{2 j}<2 A_{a j}-1$ and $\varepsilon_{j}>\zeta_{j}$, the total amount of traction torque follows the desired traction characteristic curve $T^{*}$ within finite time.

Proof. A calculation matrix is defined as $Z=\left[\begin{array}{lllll}s_{2} & x_{a 1} & x_{a 2} & \ldots & x_{a m}\end{array}\right]^{\mathrm{T}}$.

The positive Lyapunov function is selected as

$$
V_{2}=\frac{1}{2} Z^{T} Z
$$

Using equations (14) and (18) and substituting them in equation (20) yield the following:

$$
\begin{aligned}
\dot{V}_{2}= & Z^{T} \dot{Z} \\
= & s_{2} \sum_{j=1}^{m}\left[-\bar{J}_{\mathrm{eq} j} \bar{a}_{0 j} x_{1 j}+\left(\bar{b}_{\mathrm{eq} j}-\bar{J}_{\mathrm{eq} j} \bar{a}_{1 j}\right) x_{2 j}+\bar{J}_{\mathrm{eq} j} \bar{b}_{j} u_{j}+d_{2 j}\right] \\
& -s_{2} \dot{T}^{*}+\sum_{j=1}^{m}\left(x_{a j} \dot{x}_{a j}\right) \\
= & -s_{2} \dot{T}^{*}+\sum_{j=1}^{m}\left[-\bar{J}_{\mathrm{eq} j} \bar{a}_{0 j} x_{1 j} s_{2}+\left(\bar{b}_{\mathrm{eq} j}-\bar{J}_{\mathrm{eq} j} \bar{a}_{1 j}\right) x_{2 j} s_{2}\right. \\
& +s_{2} \bar{J}_{\mathrm{eq} j} \bar{b}_{j} \Delta u+s_{2} \bar{J}_{\mathrm{eq} j} \bar{b}_{j} v_{j}+d_{2 j} s_{2}-A_{a j} x_{a j}^{2} \\
& \left.-\frac{\left|s_{2} B_{a j} \Delta u\right|+0.5 \Delta u^{2}}{\left|x_{a j}\right|} x_{a j}+x_{a j} \Delta u\right] .
\end{aligned}
$$

By substituting controller equation (19) and observed error equation (13) and by using an inequality of the type $x y \leq 0.5 x^{2}+0.5 y^{2}$, we can obtain the following:

$$
\begin{aligned}
\dot{V}_{2} \leq & \sum_{j=1}^{m}\left[-s_{2}^{2} c_{2 j}+0.5 c_{2 j} s_{2}^{2}+0.5 c_{2} x_{a j}^{2}-A_{a j} x_{a j}^{2}\right. \\
& -\left|s_{2} B_{a j} \Delta u\right|+s_{2} \bar{J}_{\mathrm{eq} j} \bar{b}_{j} \Delta u-0.5 \Delta u^{2}+0.5 x_{a j}^{2}+0.5 \Delta u^{2} \\
& \left.+\left|s_{2}\right|\left|d_{2 j}-\widehat{d}_{2 j}\right|-\left|s_{2}\right| \varepsilon_{j}\right] \\
\leq & \sum_{j=1}^{m}\left[-0.5 c_{2 j} s_{2}^{2}-\left(A_{a j}-0.5-0.5 c_{2 j}\right) x_{a j}^{2}-\left(\varepsilon_{j}-\zeta_{j}\right)\left|s_{2}\right|\right] \\
\leq & -\min \left\{0.5 c_{2 j}, A_{a j}-0.5-0.5 c_{2 j}\right\}\|Z\|^{2}-\sum_{j=1}^{m}\left[\left(\varepsilon_{j}-\zeta_{j}\right)\left|s_{2}\right|\right] \\
= & -\min \left\{n_{1}, n_{2}\right\}\|Z\|^{2}-\sum_{j=1}^{m}\left[\left(\varepsilon_{j}-\zeta_{j}\right)\left|s_{2}\right|\right] .
\end{aligned}
$$

If the design parameters satisfy $0<c_{2 j}<2 A_{a j}-1$ and $\varepsilon_{j}>\zeta_{j}$, then $n_{1}>0$ and $n_{2}>0$, then 
TABLE 1: Motor parameter settings.

\begin{tabular}{lcccccc}
\hline Motor & $R(\Omega)$ & $L(H)$ & $b_{\text {eq }}(\mathrm{N} \cdot \mathrm{m} \cdot \mathrm{s})$ & $J_{\mathrm{eq}}\left(\mathrm{kg} \cdot \mathrm{m}^{2}\right)$ & $k_{m}(\mathrm{mN} \cdot \mathrm{m} / \mathrm{A})$ & $k_{e}(\mathrm{mv} / \mathrm{rad} / \mathrm{s})$ \\
\hline 1 & 2.5 & 0.612 & 0.08 & 2.4 & 82.2 & 82.3215 \\
2 & 2.2 & 0.55 & 0.06 & 2.3 & 81.5 & 82.2 \\
3 & 2.3 & 0.6 & 0.07 & 2.35 & 81.5 & 7.888 \\
4 & 2.4 & 0.58 & 0.075 & 2.15 & 82 & 7.9 \\
\hline
\end{tabular}

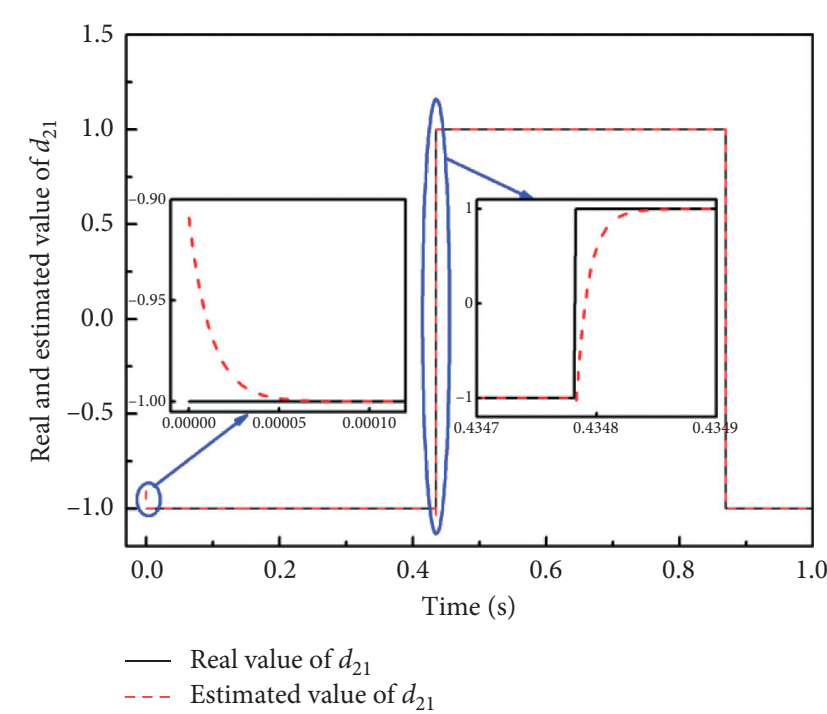

(a)

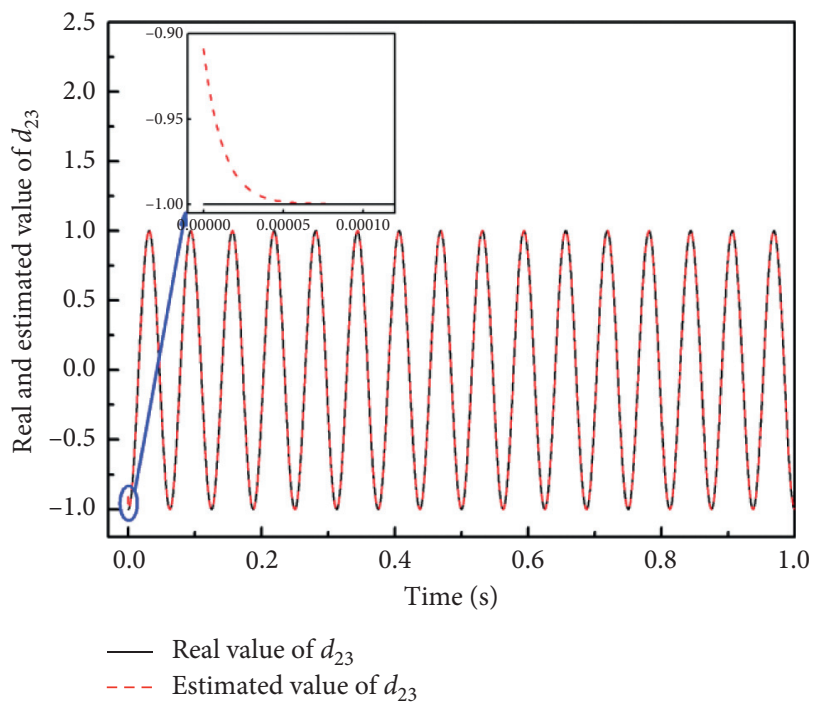

(c)

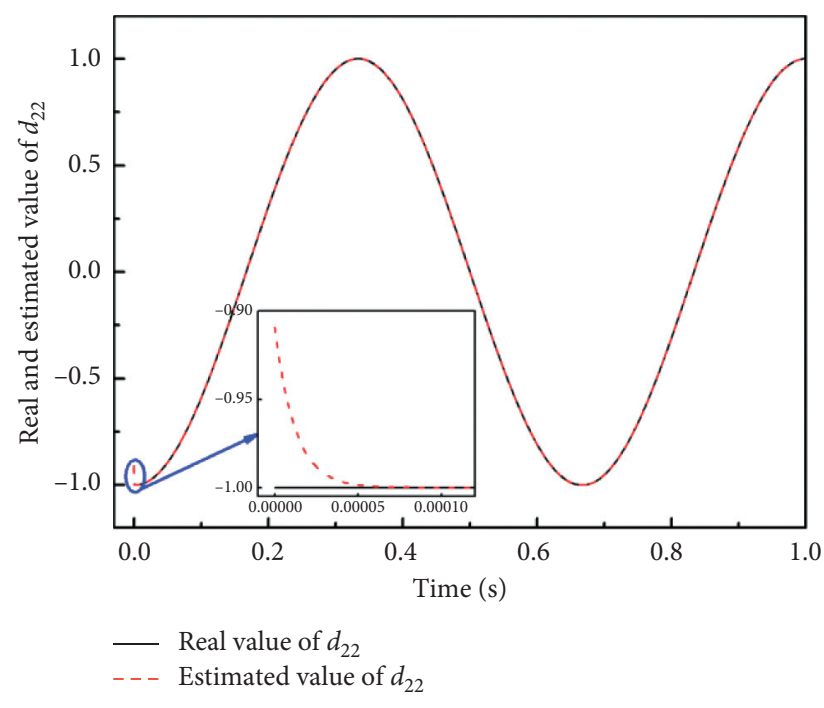

(b)

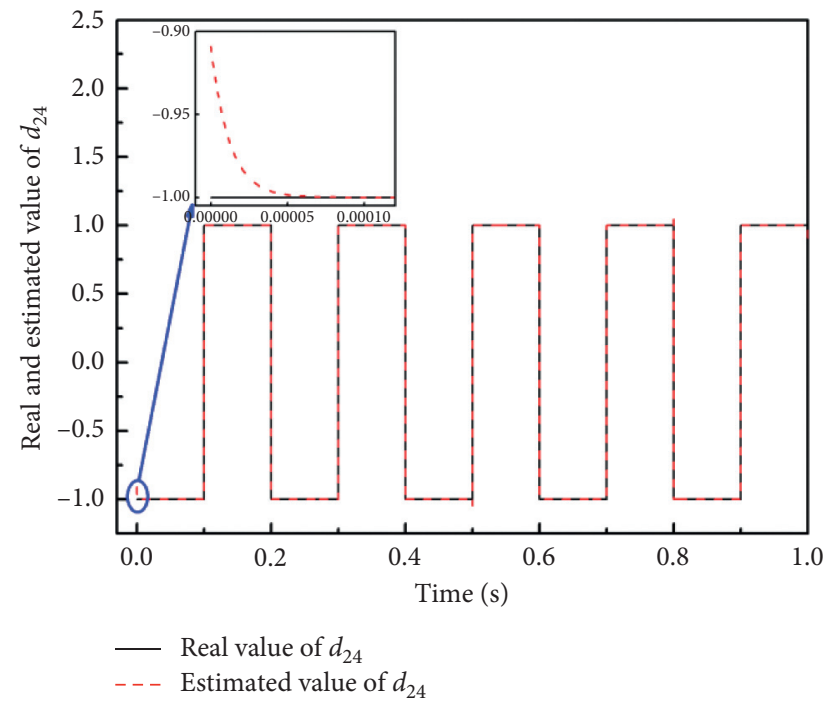

(d)

FIgURe 3: SMDO performance assessment curves: (a) fast-varying disturbance of Motor 1, (b) slow-varying disturbance of Motor 2, (c) high-frequency noise of Motor 3, (d) uniform noise of Motor 4.

$$
\dot{V}_{2} \leq-\min \left\{n_{1}, n_{2}\right\}\|Z\|^{2}=-n\|Z\|^{2}
$$

where $n=\min \left\{n_{1}, n_{2}\right\}$. Therefore, $\dot{V}_{2} \leq 0$ is established; that is, the system is asymptotically stable, and the switching surface is reached and maintained within a finite time $T$. In other words, when $t>T, s_{2} \longrightarrow 0$ and $x_{a j} \longrightarrow 0$. Given that the selected sliding-mode surface is $s_{2}=e, e \longrightarrow 0$ can be obtained. Given that the designed error is $e=\sum_{j=1}^{m}\left(x_{3 j}\right)-T^{*}$, then $\sum_{j=1}^{m}\left(x_{3 j}\right)=T^{*}$.

\section{Simulations Results}

Matlab/Simulink simulation software was used to verify the designed observer and controller; simultaneously, the RTLab semiphysical experiment platform was used to verify the 


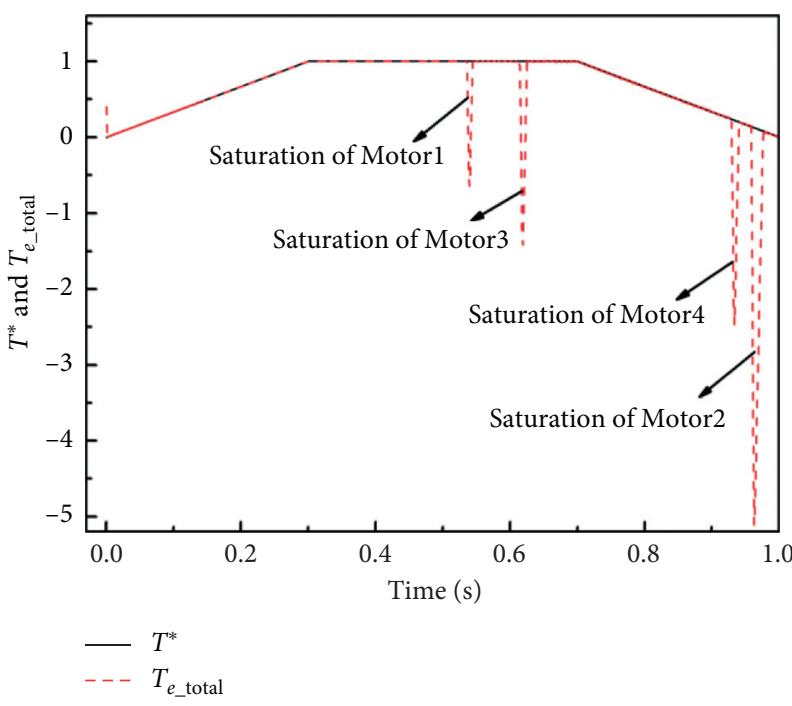

(a)

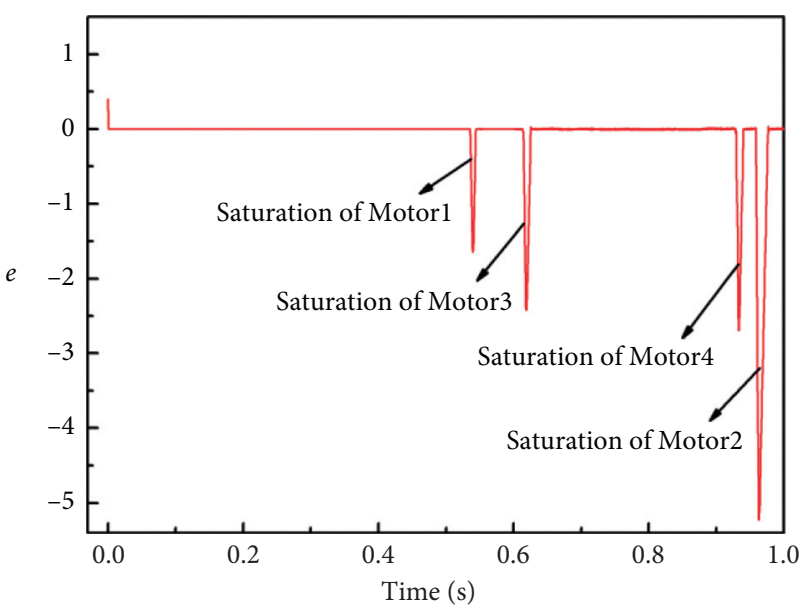

(b)

FIgURE 4: The TACTC without AW: (a) the tracking curve of total traction torque amount, (b) the tracking error curve of total traction torque amount.

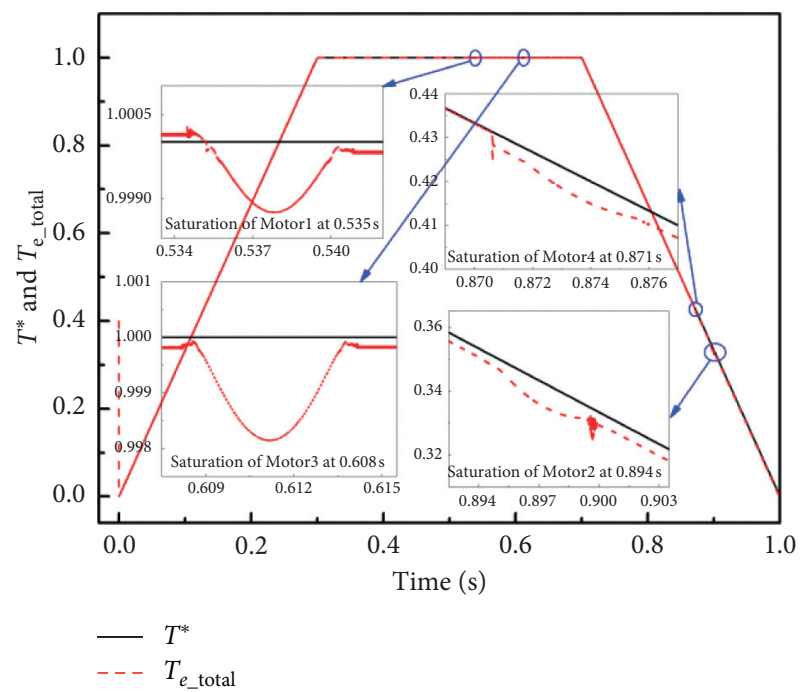

(a)

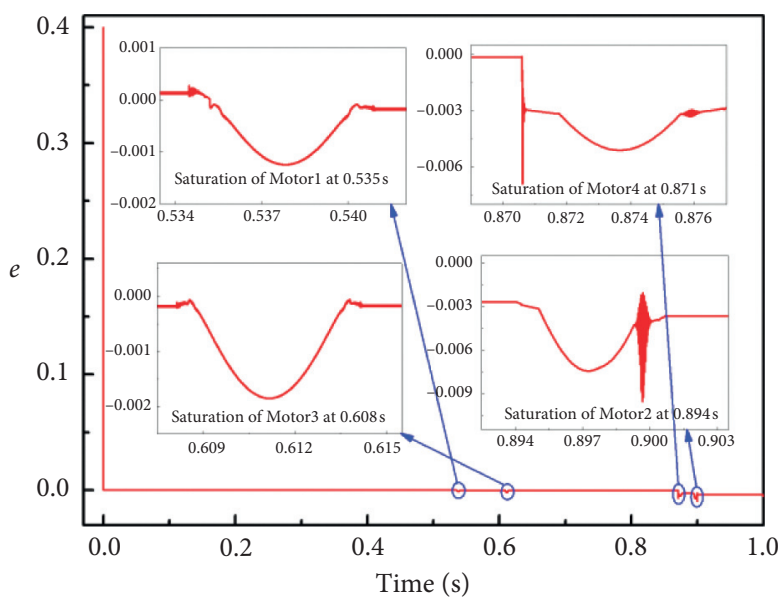

(b)

FIgURE 5: The TACTC with AW: (a) the tracking curve of total traction torque, (b) the tracking error curve of total traction torque.

practicability of the control strategy proposed herein. A multimotor traction system consisting of four motors with different parameters was used as a simulation object. Table 1 lists the parameter settings for each individual motor. The initial torque of each motor was set as 0.1.

In controller equation (19), the sliding-mode parameters $\varepsilon_{j}$ of each motor were set as 100 . The desired traction characteristic curve was composed of a piecewise function: the motor acceleration phase $(0 \longrightarrow 0.3 \mathrm{~s})$, constant-speed motor operation phase $(0.3 \longrightarrow 0.7 \mathrm{~s})$, and motor-braking phase $(0.7-1 \mathrm{~s})$. These phases are indicated in equation (24).

$$
T^{*}= \begin{cases}\frac{10}{3} t, & t<0.3, \\ 1, & 0.3 \leq t \leq 0.7, \\ -\frac{10}{3} t+\frac{10}{3}, & 0.7<t \leq 1 .\end{cases}
$$

5.1. SMDO Performance Simulation. Four disturbance signals, namely, a fast-varying disturbance, slow-varying 

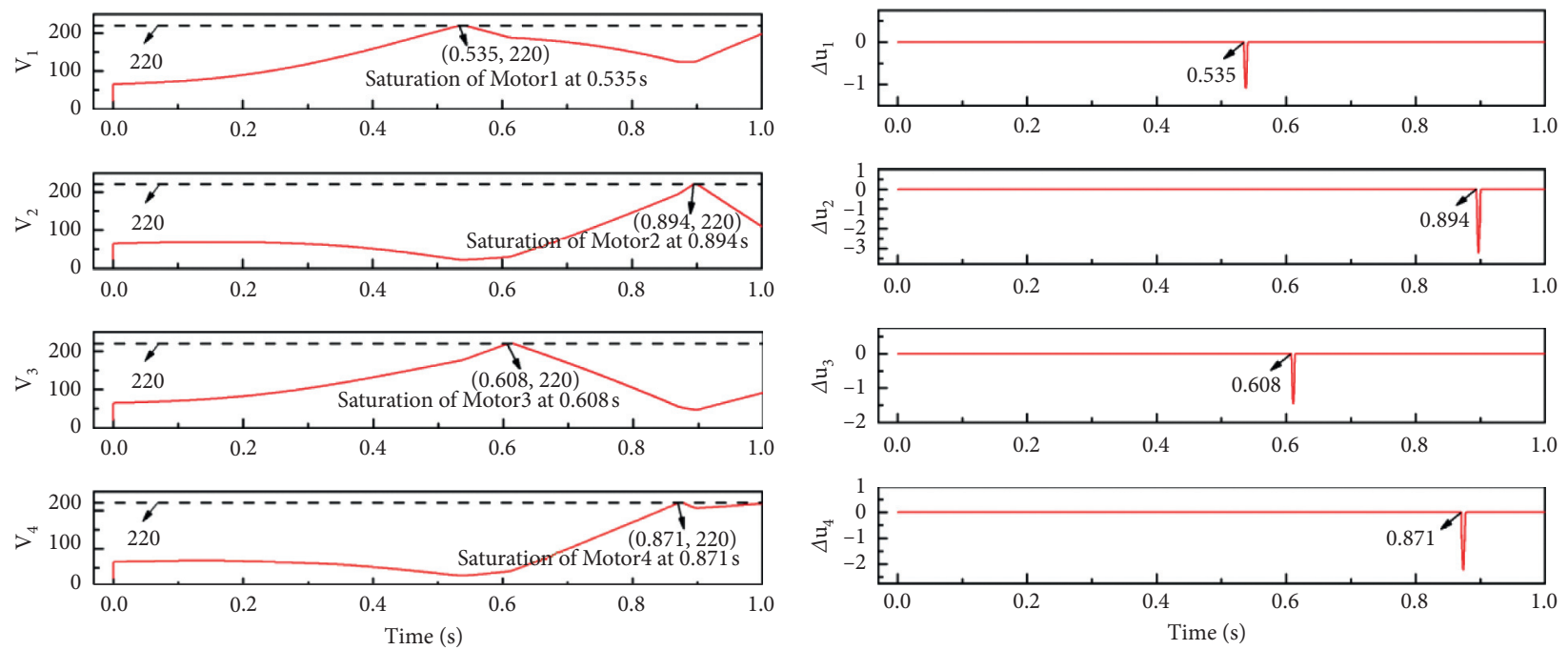

(a)

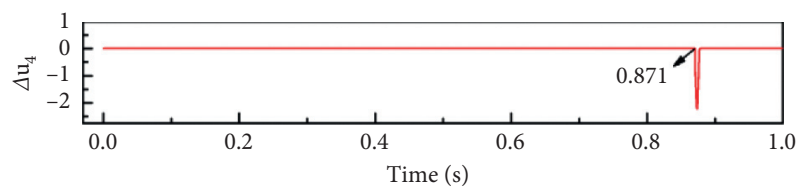

(b)

FIgURE 6: Input curve of the TACTC with AW: (a) the control input curve, (b) the saturated input error curve.
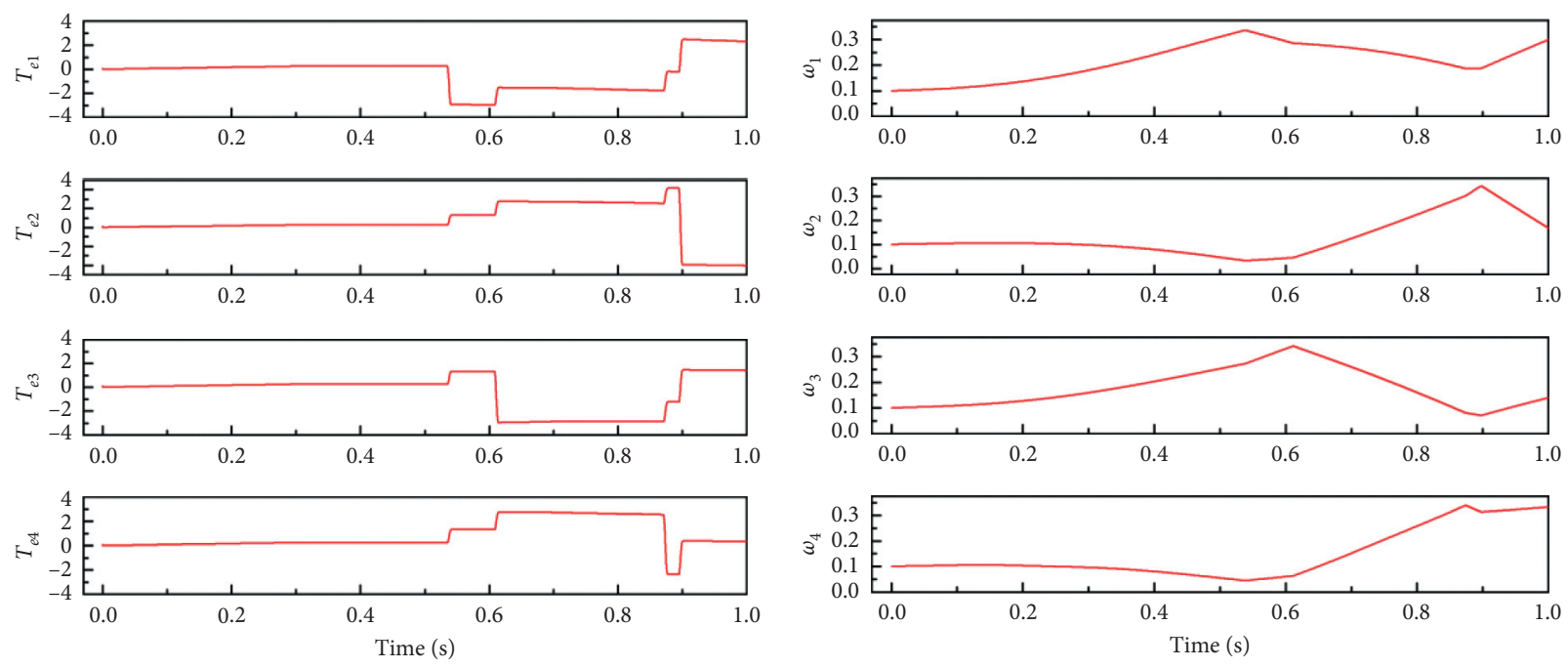

(a)

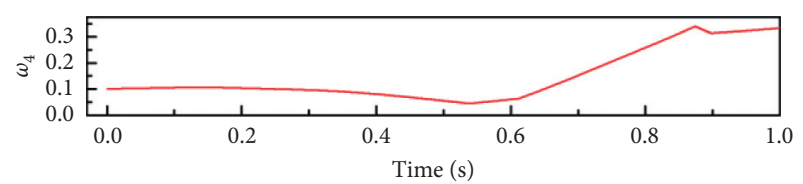

(b)

FIgURE 7: Output curve of the TACTC with AW: (a) the output torque curve, (b) the output angular velocity curve.

disturbance, high-frequency noise, and uniform noise, were applied to the four motors to verify the SMDO performance. In equation (5), the parameters were set as follows: $k_{1}=1$ and $k_{3}=10$. The observed curves of the four types of disturbances are shown in Figure 3.

The observed curves of the four disturbance signals (Figure 3) show that the maximum tracking time of the observer is approximately $0.00007 \mathrm{~s}$. Although the fastvarying disturbance in Figure 3(a) has errors, the tracking can be completed within the maximum tracking time. Therefore, the designed SMDO has excellent observation performance and satisfies the design requirements of an actual controller.
5.2. TACTC Performance Simulation. By combining the basic ideas reported in previous works $[1,2]$, a comparative simulation of the TACTC with and without AW is performed to verify that the proposed control strategy can greatly improve the TACTC performance and solve the input saturation problem of multiple motors.

The early results showed that the consensus-based totalamount cooperative tracking control approach is effective for reducing tracking time and tracking errors. However, multimotor coordination often causes saturation problems when the input value for a motor often exceeds its maximum allowable input value. Therefore, in the simulation, the input saturation value was set as \pm 220 ; the TACTC without AW 
can obtain the tracking curve and the tracking error curve of total traction torque amount (Figure 4).

In Figure 4(a), the desired traction characteristic curve is indicated by a solid black line, and the total traction torque amount of all the motors is indicated by a red dashed line. In Figure 4(b), the tracking error curve of the total traction torque amount is represented by a solid red line. As shown in Figure 4, multimotor coordination causes the four motors to be saturated at different times. The TACTC ensures that the total torque traction amount agrees with the desired traction characteristic curve; however, input saturation results in large and increasingly serious fluctuations of the total torque traction amount. Therefore, when input saturation occurs, the TACTC without AW seriously reduces the overall traction performance.

For the auxiliary AW system and controller, represented by equations (14) and (19), respectively, the parameters are set as follows: $A_{a j}=2 \times 10^{6}, B_{a j}=1500, c_{2 j}=1.6 \times 10^{5}$, and $\tau=0.00001$. The TACTC with AW realizes the tracking curve and the tracking error curve of total traction torque amount, as shown in Figure 5.

As shown in Figure 5(a), the total traction torque amount of all the motors and desired traction characteristic curve are indicated by a dashed red line and solid black line, respectively. As shown in Figure 5(b), the tracking error curve of total traction torque amount is represented by a solid red line. In Figure 5, the input saturation problems of the four motors at $0.535,0.894,0.608$, and 0.871 s are shown. The curves in Figures 5(a) and 5(b) show that the proposed TACTC with AW greatly improves the influence of input saturation on the overall tracking performance. The overall tracking performance can be guaranteed even in the case that two motors are saturated in the constant-speed operation phase and the braking phase. To show the effectiveness of the TACTC with AW, the input and output curves shown in Figures 6 and 7 were obtained.

As shown in Figures 6 and 7, the corresponding curve in each figure and the input saturation curve are indicated by a solid red line and dashed black line, respectively. The curve Figure 6(a) shows that the control inputs of each motor are controlled effectively below the rated voltage of $220 \mathrm{~V}$. The saturated input error curve shown in Figure 6(b) shows the saturation time of each motor and the timeliness of AW. The output torque and angular velocity curve of Figure 7 suggests that the multimotor system ensures that the total traction torque obtained by coordinating the traction torque of the motors before and after saturation is in agreement. Therefore, compared with the existing total-amount coordinated tracking control strategy, the proposed control strategy can effectively ensure the overall traction performance of the locomotive even under the control input saturation condition in the multimotor coordination process.

5.3. RT-Lab Semiphysical Experiment. The effectiveness of the proposed control strategy was verified by using the RTLab semiphysical experiment platform. This platform is

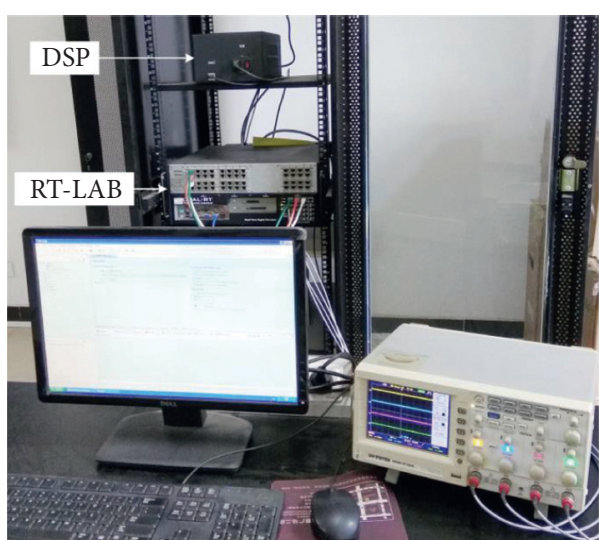

Figure 8: RT-Lab semiphysical experiment platform.

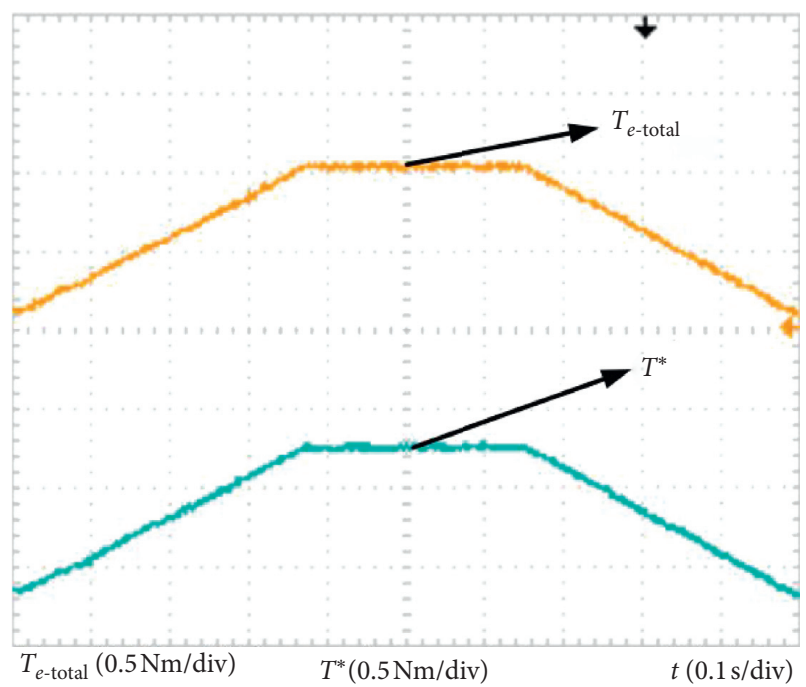

FIgURE 9: The tracking effect curve.

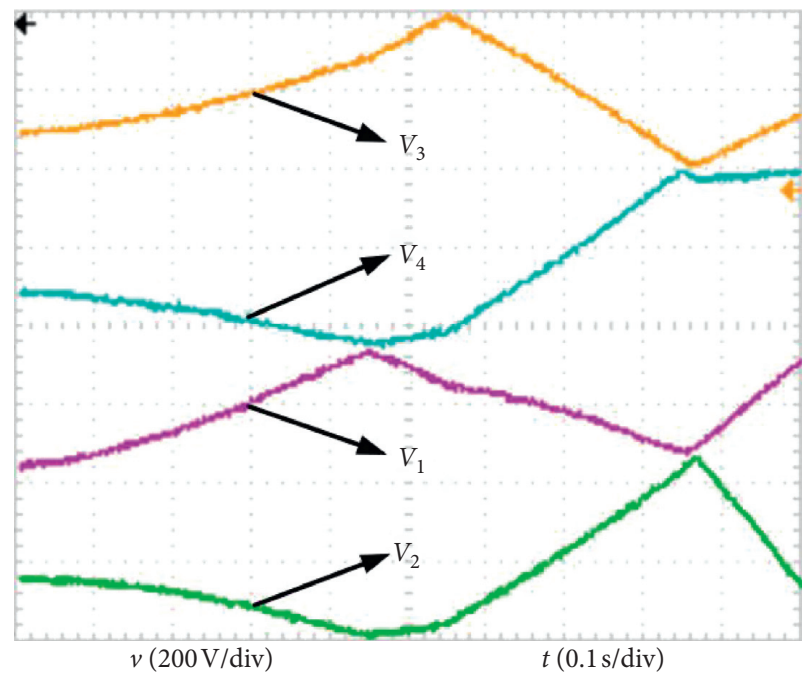

Figure 10: The control input curve. 
equipped with TMS320F2812 digital signal processor (DSP), RT-Lab OP5600 simulation motor, connecting lines, etc. The platform is shown in Figure 8. The experimental results are shown in Figures 9 and 10.

Figure 9 shows the tracking effect curve of the total traction torque of the total amount coordinated control strategy. The yellow waveform of channel 1 represents the sum of the output torques of all motors, and the blue waveform of channel 2 represents the desired traction characteristic curve. The sum of the traction torques of all motors agrees well with the desired traction characteristic curve. Thus, good overall traction performance of the locomotive is ensured. The control input curve of each motor is shown in Figure 10; the inputs of each motor are effectively controlled below the input saturation level of $220 \mathrm{~V}$. In summary, Figures 9 and 10 show that the experimental results are consistent with the simulation results obtained using Simulink. Thus, the proposed control strategy effectively solves the input saturation problem in multimotor total amount coordination and provides a theoretical basis for practical engineering applications.

\section{Conclusions}

The designed control algorithm extends the multimotor coordinated control from the consensus of the individual to the consensus of the total amount of traction torque. Given the severity and complexity of the input saturation problem of multiple motors under the consensus of the total amount of traction torque, a TACTC strategy that combines the SMDO and auxiliary AW technology was developed. First, the observed values of unknown compound disturbances were introduced into the controller design. Consequently, the phenomenon of cancellation of disturbances by increasing the sliding-mode switching gain in sliding-mode control was avoided. Next, the state of the auxiliary AW system was introduced into the controller design. Thus, the influence of input saturation on the tracking performance under the consensus of the total amount of traction torque was reduced. The proposed TACTC strategy was suitable not only for the single-motor input saturation, but also for the input saturation of multiple motors in a multimotor traction system. The proposed strategy was validated by Matlab/ simulink simulation and RT-Lab semiphysical experiment. In future work, further optimization in terms of the speed of the auxiliary AW system can be considered.

\section{Data Availability}

The model data of Matlab used to support the findings of this study are available from the corresponding author upon request.

\section{Conflicts of Interest}

The authors declare that there are no conflicts of interest regarding the publication of this paper.

\section{Acknowledgments}

This research was supported by the Natural Science Foundation of China (nos. U1934219, 61773159, and 61473117), Excellent Youth Research Project of Education Department of Hunan Province (no. 18B303), Project of Hunan Provincial Department of Education (no. 19A137), and Hunan Provincial Natural Science Foundation of China (no. 2020JJ6083).

\section{References}

[1] C. Zhang, Z. Lin, J. Liu, J. He, H. Wu, and P. Li, "Consensusbased total-amount cooperative tracking control for multimotor locomotive traction system," Journal of the Franklin Institute, vol. 356, no. 2, pp. 819-834, 2019.

[2] C. Zhang, Z. Lin, S. X. Yang, and J. He, "Total-amount synchronous control based on terminal sliding-mode control," IEEE Access, vol. 5, pp. 5436-5444, 2017.

[3] M. Brenna, A. Capasso, M. C. Falvo, F. Foiadelli, R. Lamedica, and D. Zaninelli, "Investigation of resonance phenomena in high speed railway supply systems: theoretical and experimental analysis," Electric Power Systems Research, vol. 81, no. 10, pp. 1915-1923, 2011.

[4] S. Mobayen and G. Pujol-Vázquez, "A robust LMI approach on nonlinear feedback stabilization of continuous state-delay systems with lipschitzian nonlinearities: experimental validation," Iranian Journal of Science and Technology, Transactions of Mechanical Engineering, vol. 43, no. 3, pp. 549-558, 2018.

[5] M. Afshari, S. Mobayen, R. Hajmohammadi et al., "Global sliding mode control via linear matrix inequality approach for uncertain chaotic systems with input nonlinearities and multiple delays," Journal of Computational and Nonlinear Dynamics, vol. 13, no. 3, pp. 1-34, 2018.

[6] J. Lozier, "A steady state approach to the theory of saturable servo systems," IRE Transactions on Automatic Control, vol. 1, no. 1, pp. 19-39, 1956.

[7] M. V. Kothare, P. J. Campo, M. Morari, and C. N. Nett, "A unified framework for the study of anti-windup designs," Automatica, vol. 30, no. 12, pp. 1869-1883, 1994.

[8] S. Tarbouriech and M. Turner, "Anti-windup design: an overview of some recent advances and open problems," IET Control Theory \& Applications, vol. 3, no. 1, pp. 1-19, 2009.

[9] S. Galeani, S. Tarbouriech, M. Turner et al., "A tutorial on modern anti-windup design," European Journal of Control, vol. 15, no. 3-4, pp. 418-440, 2009.

[10] M. Hussain, M. Rehan, C. K. Ahn, and Z. Zheng, "Static antiwindup compensator design for nonlinear time-delay systems subjected to input saturation," Nonlinear Dynamics, vol. 95, no. 3, pp. 1879-1901, 2018.

[11] M. C. Turner and M. Kerr, "A nonlinear modification for improving dynamic anti-windup compensation," European Journal of Control, vol. 41, pp. 44-52, 2018.

[12] L. Yu, R. Wang, W. Xia, and W. Wang, "An anti-windup method for a class of uncertain MIMO systems subject to actuator saturation with LADRC," Information Sciences, vol. 462, pp. 417-429, 2018.

[13] O. Lamrabet, E. H. Tissir, and F. El Haoussi, "Anti-windup compensator synthesis for sampled-data delay systems," Circuits, Systems, and Signal Processing, vol. 38, no. 5, pp. 2055-2071, 2019. 
[14] H. Zhu, G. Qin, Y. Yan et al., "Delayed antiwindup control using a decoupling structure," Mathematical Problems in Engineering, vol. 2013, Article ID 248153, 7 pages, 2013.

[15] H.-T. Chen, S.-M. Song, and Z.-B. Zhu, "Robust finite-time attitude tracking control of rigid spacecraft under actuator saturation," International Journal of Control, Automation and Systems, vol. 16, no. 1, pp. 1-15, 2018.

[16] B. Ye, L. Long, and J. Zhao, "Adaptive dynamic surface control of switched MIMO nonlinear systems with input saturation and its application to NSVs," Asian Journal of Control, vol. 4, pp. 1-14, 2019.

[17] M. Yan, Y. Tang, P. Yang, and L. Zuo, "Consensus based platoon algorithm for velocity-measurement-absent vehicles with actuator saturation," Journal of Advanced Transportation, vol. 2017, Article ID 8023018, 8 pages, 2017.

[18] Q. Guo, J. Yin, T. Yu, and D. Jiang, "Saturated adaptive control of an electrohydraulic actuator with parametric uncertainty and load disturbance," IEEE Transactions on Industrial Electronics, vol. 64, no. 10, pp. 7930-7941, 2017.

[19] S. Kiamini, A. Jalilvand, and S. Mobayen, "LMI-based robust control of floating tension-leg platforms with uncertainties and time-delays in offshore wind turbines via T-S fuzzy approach," Ocean Engineering, vol. 154, no. 15, pp. 367-374, 2018.

[20] M. Jafari and S. Mobayen, "Second-order sliding set design for a class of uncertain nonlinear systems with disturbances: an LMI approach," Mathematics and Computers in Simulation, vol. 156, pp. 110-125, 2019.

[21] M. Hussain, M. Rehan, and M. Tufail, "Robust antiwindup for one-sided Lipschitz systems subject to input saturation and applications," IEEE Transactions on Industrial Electronics, vol. 65 , no. 12, pp. 9706-9716, 2018.

[22] G. Peng, C. Yang, W. He et al., "Force sensorless admittance control with neural learning for robots with actuator saturation," IEEE Transactions on Industrial Electronics, vol. 99, pp. 1-10, 2019.

[23] R. Bai, "Adaptive sliding-mode control of automotive electronic throttle in the presence of input saturation constraint," IEEE/CAA Journal of Automatica Sinica, vol. 5, no. 4, pp. 878-884, 2018.

[24] J. Lopez-Gomez, M. A. D. Vargas-Treviño, S. Vergara-Limon et al., "Influence of PWM torque control frequency in DC motors by means of an optimum design method," IEEE Access, vol. 8, pp. 80691-80706, 2020.

[25] F. E. Hoyos, J. E. Candelo-Becerra, and C. I. Hoyos Velasco, "Application of zero average dynamics and fixed point induction control techniques to control the speed of a DC motor with a buck converter," Applied Sciences, vol. 10, no. 5, pp. 1807-1819, 2020.

[26] M. S. Widyan and A. M. Harb, "Dynamical and operational performance of shunt and permanent-magnet DC motors powered by lead-acid batteries for pumping applications," Nonlinear Dynamics, vol. 99, no. 1, pp. 341-349, 2020.

[27] F. Beltran-Carbajal, A. Valderrabano-Gonzalez, J. C. RosasCaro, and A. Favela-Contreras, "An asymptotic differentiation approach of signals in velocity tracking control of DC motors," Electric Power Systems Research, vol. 122, pp. 218223, 2015.

[28] G. Sulligoi, D. Bosich, L. Zhu et al., "Linearizing control of shipboard multi-machine MVDC power systems feeding constant power loads," in Proceedings of the 2012 IEEE Energy Conversion Congress and Exposition (ECCE), pp. 691-697, Raleigh, NC, USA, September 2012.
[29] G. Sulligoi and R. C. Kavanagh, "An innovative method for improved real-time measurements of angular acceleration in motion control systems," in Proceedings of the International Symposium on Power Electronics, Electrical Drives, Automation and Motion, pp. 658-662, Taormina, Italy, May 2006.

[30] F. Beltran-Carbajal, A. Valderrabano-Gonzalez, and J. C. Rosas-Caro, "Output feedback control for robust tracking of position trajectories for DC electric motors," Electric Power Systems Research, vol. 107, no. 2, pp. 183-189, 2014.

[31] J. He, X. Chen, S. Mao, C. Zhang, and J. Liu, "Virtual line shafting-based total-amount coordinated control of multimotor traction power," Journal of Advanced Transportation, vol. 2020, Article ID 4735397, 9 pages, 2020.

[32] A. F. Filippov, Differential Equations with Discontinuous Righthand Sides: Control Systems, Springer, Berlin, Germany, 2013.

[33] Y. Shtessel, C. Edwards, L. Fridman et al., Sliding Mode Control and Observation, Springer, Berlin, Germany, 2014.

[34] W. He, Y. Dong, and C. Sun, "Adaptive neural impedance control of a robotic manipulator with input saturation," IEEE Transactions on Systems Man \& Cybernetics Systems, vol. 46, no. 3, pp. 334-344, 2017. 